\title{
Atención en psicopediatría para el manejo de emociones en los niños durante la pandemia COVID-19
}

\author{
Serdán Ruiz David Leonardo \\ ORCID: https://orcid.org/0000-0001-5134-9921 \\ drserdanruiz@hotmail.com \\ Hospital Básico Dr. Eduardo Montenegro \\ Chillanes, Ecuador
}

\author{
Vásquez Bone Katterine Kariuxy \\ ORCID: https://orcid.org/0000-0002-5094-1760 \\ kvasquez@utb.edu.ec \\ Universidad Técnica de Babahoyo \\ Babahoyo,Ecuador
}

\author{
Yupa Pallchisaca Ana Emperatriz \\ ORCID: https://orcid.org/0000-0002-2397-9026 \\ ayupap@utb.edu.ec \\ Universidad Técnica de Babahoyo \\ Babahoyo, Ecuador
}

Recibido (26/04/21), Aceptado (14/05/21)

\begin{abstract}
Resumen: La pandemia de la COVID 19 afectó la salud mental, además de la física, especialmente de los sectores más vulnerables desde el punto de vista socioeconómico, entre los cuales destacan los niños y niñas. A ello se agregan los efectos psicológicos de las medidas de emergencia que se han tomado para afrontar la pandemia, que han agudizado los problemas de salud mental de la familia: cierre de las escuelas, confinamiento, entre otras. Las organizaciones internacionales han dictado lineamientos generales para atender esta emergencia en la salud mental de los niños y niñas combinando el servicio de salud mental y el apoyo psicosocial. En este artículo, mediante una revisión documental, se sistematizan los análisis de la situación de la salud mental entre los niños, y sobre algunas acciones preventivas, orientadas a lograr el manejo del stress infantil, el control de las emociones y el desarrollo de la resiliencia.
\end{abstract}

Palabras Clave: COVID 19, salud mental, apoyo psicosocial, sectores vulnerables, resiliencia

\section{Attention in psychopediatry for the management of emotions in children during the COVID-19 pandemic}

\begin{abstract}
The COVID 19 pandemic affected mental health, as well as physical health, especially in the most socioeconomic vulnerable sectors, including children. In addition, the psychological effects of the emergency measures taken to address the pandemic have exacerbated the mental health problems of the family: School closure, confinement, among others. International organizations have issued general guidelines for addressing this emergency in the mental health of children by combining mental health service and psychosocial support. This article, through a documentary review, systematizes analyzes of the mental health situation among children, and some preventive actions aimed at achieving the management of childhood stress, the control of emotions and the development of resilience.
\end{abstract}

Keywords:COVID 19, mental health, psychosocial support, vulnerable sectors, resilience.

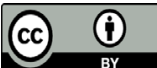




\section{Introducción}

Los niños y niñas constituyen uno de los segmentos de la población más afectados por la pandemia de la COVID 19, no sólo por el peligro de la infección y sus consecuencias físicas, sino también por el impacto en su salud mental. Ellos además sufren las consecuencias de las medidas profilácticas que han implementado los gobiernos, tales como el cierre de las escuelas y sus sistemas de apoyo, al igual que el confinamiento el cual puede contribuir a agudizar problemas de salud mental preexistentes en las familias.

Por tal motivo, las organizaciones internacionales, tales como la OMS (Organización Mundial de la Salud) y la UNICEF (Organización de las Naciones Unidas para la Infancia y la Juventud) han dado orientaciones a los gobiernos para realizar una atención psicopediátrica dirigida a los niños, niñas y adolescentes, que combine las características del servicio de salud mental con el del apoyo psicosocial, con base en organizaciones comunales y locales.

En este artículo se realizará una revisión documental con el objetivo de recoger y sistematizar información de contenido teórico y empírico, de fuentes científicas e institucionales internacionales, acerca de las consecuencias de la pandemia de la COVID 19 en la salud mental de la población infantil, la descripción de las características de esos impactos, los derechos específicos de los niños que deben ser garantizados por una adecuada política sanitaria en tiempos de pandemia, así como las políticas propuestas como respuestas desde el punto de vista psicopediátrico, orientadas por los lineamientos de las organizaciones internacionales y los gobiernos.

Se realizará una exploración y revisión documental y se discutirán los conceptos, explicaciones y datos suministrados por esas fuentes. Posteriormente se elaborarán unas conclusiones que invitarán a continuar la investigación sobre el tema. Por último, se informará de las referencias bibliográficas utilizadas.

\section{Desarrollo}

\section{A.Los niños y la salud mental}

El derecho a la salud y a la salud mental, específicamente de los niños, niñas y adolescentes, ha sido consagrado por diversos documentos de validez internacional, como la Convención de los Derechos de la Infancia [1]. Justifican esta declaración, entre otras argumentaciones, los datos preocupantes acerca de la salud mental en este segmento de la población mundial, que sufre con más fuerza los impactos de las diversas catástrofes y conflictos, así como en la actualidad, los de la pandemia por COVID 19.

La salud mental comprende el bienestar emocional, psicológico y social y se refiere a los pensamientos, los sentimientos y el comportamiento. Su logro depende de algunas líneas de acción entre las que son determinantes las estrategias que se desarrollan frente al estrés, ante los demás y la manera como se toman decisiones. La OMS la considera un componente fundamental de la salud en general, entendida como un estado de bienestar físico, mental y social, y no solamente la ausencia de enfermedades. La salud mental de niños, niñas y adolescentes incluye: el bienestar personal (pensamientos positivos: optimismo, calma, autoestima, confianza en sí mismos), bienestar interpersonal (relaciones con los demás, cuidado responsable y sensible, sentido de pertenencia y la capacidad de estar cerca de los demás) y las capacidades y conocimientos (capacidad de aprender, de tomar decisiones, de responder a los retos de la vida y expresarse) [2].

De acuerdo a los datos de los organismos internacionales, alrededor del 15\% de los niños del mundo sufren por alguna consecuencia de enfermedades mentales. En general se considera que la mitad de los problemas de salud mental comienzan a partir de los catorce años. Así mismo, se sabe que el suicidio es la segunda causa de mortalidad en jóvenes de entre 15 y 24 años. En la mayor parte de los países, los niños de los entornos sociales más desfavorecidos sufren de trastornos emocionales más pronunciados. Pero, además, los estudios indican que uno de cada cuatro niños que han sufrido aislamiento por la COVID 19, presentan síntomas de depresión o ansiedad [3].

Las afecciones en la salud mental de los niños van desde los trastornos de sueño, sentimientos de ansiedad y depresión, autolesiones y dificultades de concentración. Igualmente, se nota miedo a la soledad y a la oscuridad, pesadillas, regresión, dificultades con la alimentación, aumento de rabietas o conducta aferrada. En primaria, son más frecuentes la irritabilidad, las pesadillas, los problemas de sueño y alimentación, los dolores de cabeza y de barriga, los problemas de conducta, el apego excesivo, la competencia entre hermanos por la atención de los padres y el retraimiento social de los demás.

La experiencia histórica muestra que, al producirse pandemias infecciosas, se produce un aumento de la sintomatología ansiosa, depresiva y postraumática en la población infanto-juvenil. También se ha observado que el confinamiento tiene repercusiones negativas sobre su salud mental y física. El desarrollo de los menores se puede ver afectado por el cierre de las escuelas, la limitación de las relaciones con iguales, la imposibilidad de realizar actividad física en el exterior y la pérdida de hábitos saludables de vida [4].

La pandemia de COVID-19 se asocia con un incremento de factores de riesgo psicosociales, como son el aislamiento y la violencia intrafamiliar, la pobreza, el hacinamiento y el abuso de nuevas tecnologías. A la amenaza de la infección, se agregan los efectos de las medidas sanitarias que se han tomado en todo el mundo, tales como el cierre de las escuelas, el confinamiento prolongado, incluso los problemas de las familias para garantizar el sustento debido a la crisis económica ocasionada por la pandemia son todos factores estresores, tanto para los adultos, como para los niños. El estrés se manifiesta de diversas formas, muchas de ellas con sintomatología física. Es por eso que actualmente hay altos índices de niños con colitis nerviosa, gastritis o incluso operaciones por apendicitis.

El estrés es una respuesta inespecífica del organismo frente a las situaciones que le toca enfrentar el individuo. Es la 
condición resultante cuando las transacciones entre la persona y el medio conducen un individuo a la percepción de una discrepancia, real o ficticia, entre las demandas de la situación y los recursos biológicos o sociales de los que no disponen. Puede ser normal o extraordinaria. En todos los casos, se producen cambios neuroendocrinos que desafían al habitual homeostasis que mantiene tranquilo al organismo. Uno de esos cambios endocrinos es la producción de adrenalina cuyos efectos son: aumento del ritmo de las palpitaciones del corazón, aceleración de la producción de glucosa, El organismo responde de acuerdo con la proporción del estrés. La pandemia y toda la situación aparejada, constituye un factor estresor de primera importancia en la actualidad que afecta la salud mental de los niños, niñas y adolescentes [5].

\section{B.La pandemia y sus efectos en la salud mental}

Es un hecho comprobado que la pandemia de la COVID 19 y las medidas tomadas para hacerle frente, están afectando significativamente todos los aspectos de la vida de los niños, niñas y adolescentes, tanto en el aspecto físico, su desarrollo, sus posibilidades de aprendizaje y su comportamiento, además de impactar en factores como la seguridad económica de sus familias y la protección frente al posible abuso y violencias en su contra, todo lo cual constituyen graves amenazas a su salud mental.

Existen datos recientes y contundentes que indican que la actual pandemia COVID 19 ha impactado la salud mental de las personas en todo el mundo. Así lo muestran estudios empíricos en China Irán, Estados Unidos, India, Alemania, Cuba entre otros países. Algunos trastornos psiquiátricos han aumentado significativamente, tales como la ansiedad, la depresión, el insomnio y temores generalizados, especialmente en los niños, las niñas y los adolescentes [6].

El confinamiento y el aislamiento social son factores de primera importancia en la producción de síntomas de afectación en la salud mental del segmento infanto-juvenil de la población. Se ha establecido que la mayor repercusión psicológica en niños de 5 a 9 años, sin diferencias significativas en relación al sexo; con antecedentes de patología psiquiátrica y seguimiento por la especialidad, a predominio de trastorno por déficit de atención y trastornos neuróticos. La ansiedad fue la manifestación psicológica más frecuente en los primeros meses, mientras que a los tres meses fueron la hiperactividad motora y verbal, el temor a la muerte y la ansiedad somatizada. Los niños no son más que una parte orgánica de las familias, En ella, se ha observado alteraciones en su dinámica, con predominio de las manifestaciones de ansiedad y miedo y depresión, sobre todo en las madres [7].

La tasa de los trastornos mentales es más alta en personas contagiadas y en el personal que presta la atención en salud. Se han identificado indicadores de riesgo en personas del sexo femenino y que han estado en contacto con conocidos infectados. Se ha notado la presencia de estrés postraumático en personas infectadas, así como la exacerbación de las dolencias mentales en personas que ya sufrían de esas enfermedades. Por otra parte, ayuda a reducir los factores de riesgo de trastornos emocionales el manejo adecuado de la información y la sensación de control sobre la situación que muestre el individuo. La depresión y la ansiedad se presenta en jóvenes, sobre todo de sexo femenino, provenientes de clases de bajos ingresos. Se ha notado que esos síntomas se reducen en relación directa con el nivel de escolaridad [8].

Aun tomando en cuenta estos datos generales, es importante examinar cada caso en su especificidad, pues, por una parte, los niveles de estrés postraumático son cuatro veces mayores en los niños que han sido objeto de cuarentena, y por la otra, las circunstancias extraordinarias creadas por la pandemia y las medidas profilácticas tomadas en todo el mundo pueden producir respuestas emocionales extraordinarias y, por ello mismo apropiadas, por lo que ciertas actitudes y comportamientos pueden ser interpretadas como expresiones de dolor, sufrimiento o miedo enteramente motivadas, sin que sean señales de patologías declaradas. Esta consideración lleva a enfatizar la necesidad de tomar en cuenta la evolución de esos síntomas y su relación con situaciones preexistentes, así como las condiciones del entorno en lo que se refiere a lo económico, social o familiar [2].

La reacción emocional o psicológica frente a la situación creada por la pandemia no es la misma en todos los niños. Las respuestas de cada individuo dependen de una amplia variedad de factores, tales como la edad, la personalidad, y de cómo observe que responden las personas que están a su alrededor. Por otra parte, las reacciones se ven sobre todo en su comportamiento en el que puede expresarse su intranquilidad, nerviosismo, necesidad de mayor atención o contención, tristeza, excesiva preocupación por su familia o por su propia salud, y un mutismo inusual. Durante una epidemia, es común que las personas se sientan estresadas y preocupadas. Entre las respuestas psicológicas más comunes se encuentran:

-Miedo a enfermar y morir.

- Miedo a acudir a los centros sanitarios.

- Miedo a perder el trabajo y los medios de vida.

-Miedo a sentirse excluido o ser asociado con la enfermedad.

- Miedo a perder a seres queridos e impotencia por no poder protegerlos.

-Miedo a ser separados de los seres queridos durante una cuarentena.

-Rechazo al cuidado de niños y otras personas dependientes pertenecientes a otros núcleos familiares.

-Sentimientos de impotencia, aburrimiento, soledad y depresión por estar confinados y/o aislados.

-Los profesionales sanitarios pueden sufrir estresores adicionales.

En el caso de la pandemia de coronavirus en la actualidad, el miedo aumenta porque no se conocen bien las formas de transmisión, los síntomas son muy variados, y las escuelas han sido cerradas por un periodo prolongado.

El cierre de las escuelas es parte de las medidas profilácticas recomendadas internacionalmente para hacer frente a la 
extensión de la infección. Esta resolución puede ser recibida al principio por los niños, niñas y adolescentes con alivio y hasta alegría; pero la consecuencia de la falta de socialización y la interrupción del proceso del aprendizaje se convierte en un gran problema a mediano plazo. Ahora los pequeños deben permanecer en sus casas, y allí sentir, en primer lugar, que no tienen espacio suficiente para sus actividades [9].

La insatisfacción puede ser un motivo estresante para todos, pero especialmente para aquellos que son infelices con su familia. La imposibilidad de espacio y tiempo para a los recreos, para compartir con sus compañeros, la interacción social necesaria para su salud mental se ve constreñida a reducirse a los miembros de la familia. La distancia con sus amigos puede afectar de manera diferente a cada individuo. Incluso, los mayores pueden desarrollar estrategias para mantenerse en contacto con sus amistades a través de los medios electrónicos de comunicación.

En este contexto, el rol de los padres es fundamental, aunque ellos mismos pueden estar afectados por la situación, sobre todo desde el punto de vista de sus ingresos, el muy posible desempleo por el cierre de negocios y la imposibilidad de solución de los problemas diarios de manutención de la familia, aparte de los posibles conflictos interpersonales entre los miembros del grupo familiar. Las familias son generalmente asumidas como un apoyo sustancial al proceso de aprendizaje, pero exigir que los padres asuman el trabajo de educar a sus hijos en la actualidad es sumamente complicado, por el volumen de situaciones problemáticas y hasta conflictivas con que los adultos se enfrentan durante la pandemia [10].

La educación en casa puede traer algunos beneficios o paliativos al cierre de las escuelas, pero habría que considerar las diferencias sustanciales entre las familias en lo que se refiere a la cantidad de tiempo disponible para asumir esas tareas de instrucción de los hijos, las aptitudes pedagógicas de los padres, sus conocimientos, sus dificultades para ayudar a los niños en áreas que no se manejan.

Visto desde una perspectiva más abarcadora, la continuación del cierre de las escuelas aumentará las desigualdades existentes y será especialmente perjudicial para aquellos niños, niñas y adolescentes en situación más desfavorecidas. Esto repercute incluso en la alimentación de los infantes, en aquellos países donde se le garantiza el acceso de desayunos y almuerzos en la institución educativa. El cierre de las escuelas tendrá efectos indudables en lo que se refiere a ciertas áreas del conocimiento tales como las aptitudes matemáticas, lectura y escritura. En conjunto estas pérdidas tendrán repercusión en el incremento de la pobreza.

La alternativa de utilizar la internet como recurso educativo, está restringido a la porción, que casi siempre es minoritaria, de la población que tiene acceso a esas tecnologías. Además, la eficacia de esta alternativa ello tiene que ver con la calidad de las conexiones de cada país o de las regiones a lo interior de cada país. Por supuesto, en este aspecto siempre los sectores menos favorecidos y podres, son los más golpeados [11].

La obligación de quedar confinados en su casa trae con- sigo varios factores estresantes que conspiran contra la salud mental de los niños, niñas y adolescentes. Por ello, hay que resaltar la relevancia de los padres y adultos en general para lograr crear un ambiente adecuado, de protección frente al factor estresante, comprensivo y suficientemente afectivo. En esto también juegan un papel destacado los mismos maestros y profesores, así como las políticas que logren desarrollar los gobiernos hacia las familias, especialmente las desfavorecidas por la crisis económica que ha sido la consecuencia más notable, aparte del aspecto sanitario, de la crisis por la COVID 19 [12].

La hospitalización de los niños, niñas y adolescentes que han sido infectados por el virus de la COVID 19, sufren las consecuencias emocionales de su situación. Los estresores que acompañan estas experiencias son los mismos de la enfermedad: sensación de malestar o dolor, el ambiente no familiar y la presencia de extraños, la separación de padres, familiares y representantes, el estrés que le comuniquen sus acompañantes, la ruptura de la rutina habitual, la pérdida de la autonomía personal, la incertidumbre acerca de la conducta adecuada e incluso el temor a la muerte. En este sentido, es importante atender a desarrollar las capacidades de afrontamiento que pueda tener y adquirir el individuo. Las habilidades de afrontamiento son un conjunto de respuestas cognitivas, psicofisiológicas y motoras que resulten adaptativas en la situación estresante de la hospitalización. Cuando el repertorio conductual es deficitario en este tipo de habilidades, el niño muestra reacciones inadecuadas. Por ello, se recomiendan ciertas medidas de preparación para la hospitalización, facilitando información adecuada de su situación, tratando de comunicar seguridad, confianza y serenidad, instruir al niño sobre posturas para lograr la relajación, enseñar a tensar y relajar porciones de su cuerpo, emplear un lenguaje familiar y estimular al niño a que practique técnicas de relajación [13].

La exposición prolongada a miedo, preocupación, incertidumbre y otros estresores puede tener consecuencias a largo plazo para las familias, las comunidades y las personas más vulnerables, entre las que se encuentran:

-Depresión, baja autoestima, falta de confianza en sí mismo, inseguridad, etc.

- Deterioro de las relaciones sociales y de las dinámicas económicas.

-Estigmatización de las personas que han padecido la enfermedad.

-Aumento potencial de la agresividad y agresiones hacia el gobierno y los trabajadores de primera línea (sanitarios, fuerzas de seguridad, etc.).

-Aumento potencial de la agresividad y agresiones a la infancia y a las parejas (aumento de la violencia intrafamiliar $\mathrm{y}$ de género).

-Desconfianza de la información oficial.

-Recaídas de las personas con problemas de salud mental y/o adicciones [14].

Estos miedos y reacciones no siempre surgen de miedos fundados. A veces se asocian también a falta de información, 
rumores y falsos rumores.

Por el contrario, hay personas que pueden experimentar experiencias positivas, como resiliencia y orgullo por haber sido capaces de encontrar estrategias para afrontar la situación. Personas que muestran un nivel alto de altruismo y cooperación, e incluso que sienten una gran satisfacción cuando ayudan a los demás. Por ejemplo, acompañando a las personas solas por teléfono o a través de otros medios telemáticos, ofreciendo ayuda a las personas mayores para hacer las compras, o realizando gestos de ánimo a las personas aisladas o a los profesionales sanitarios [15].

El confinamiento debido a la pandemia del COVID 19, ha llevado a situaciones estresantes a los niños, niñas y adolescentes: El cierre de escuelas; la falta de la rutina habitual; la imposibilidad de esparcimiento al aire libre en compañía de amigos; el distanciamiento físico de los seres queridos; la pérdida de familiares; el desempleo y las penurias económicas familiares; la incertidumbre generalizada sobre el futuro; la exposición excesiva a noticias inquietantes y pantallas; la falta de información adaptada; la interrupción de terapias y tratamientos específicos; y la violencia y abusos en el ámbito doméstico, son algunas de las que se han producido con mayor frecuencia. De hecho, los primeros estudios realizados en China durante la pandemia COVID-19, señalaban que la pérdida de rutinas y el estrés psicosocial estaban entre los principales factores de impacto psicológico en los niños [3] [6].

Frente a las situaciones estresantes, es fundamental desarrollar la resiliencia, la cual es la capacidad de hacer frente a la adversidad y de adaptarse tras las situaciones difíciles. La resiliencia en los niños, niñas y adolescentes depende de factores personales (personalidad, genética, presencia o ausencia de algún tipo de discapacidad); factores sociales (entorno familiar, relaciones con los profesores y los amigos); y factores ambientales (acceso a servicios sociales básicos y protección, condiciones de seguridad, nivel de inclusión y sentido de pertenencia a la sociedad) [16] [17].

Es clave en la atención a la salud mental de los niños y adolescentes, conocer las estrategias de afrontamiento de situaciones estresantes. Estas muestran esfuerzos conscientes y voluntarios para regular emociones, conductas, cogniciones, psicofisiología y variables del entorno en respuesta al estrés de acontecimientos diarios o circunstancias. Esas estrategias tienen un aspecto interno, ligado a variables disposicionales, herencia, edad y sexo, y el aspecto externo, que comprende las acciones ante las demandas de la situación específica o el entorno sociocultural. Los estudios hechos a niños y niñas de entre 9 y 12 años, han mostrado que las niñas obtienen mayores puntuaciones estadísticamente significativas en las estrategias de afrontamiento de las situaciones estresantes dando una solución activa, comunicando el problema a otros, así como buscando información y guía, con una actitud positiva que les permitía concentrarse en el afrontamiento del problema. Por su parte, los niños respondían con una estrategia de conductas agresivas. Los más pequeños prefieren la estrategia de reservarse el problema [18].

Los factores asociados con las repercusiones psicosocia- les se relacionan tanto con la dimensión y los efectos de la pandemia, como con el grado de vulnerabilidad a la que se está expuesto en el contexto. En este sentido, algunos grupos sociales son más vulnerables y propensos al abandono, la exclusión y la negligencia, siendo fundamental una mayor atención de la comunidad y de las entidades públicas. Los desafíos que surgen del confinamiento en el hogar, del aislamiento social, de la disminución o ausencia de un ingreso, del apoyo social y relacional ofrecido por instituciones como escuelas y servicios que garantizan la protección de derechos y necesidades básicas, implican transformaciones profundas y nuevas organizaciones de la vida cotidiana de los niños que viven en condiciones vulnerables [10].

La atención a las personas con problemas psicológicos debe incluir estrategias sociales y económicas, además de las estrictamente relacionadas con la salud. Los grupos que viven en contextos de vulnerabilidad social están más expuestos a los riesgos derivados de la pandemia, debido a problemas estructurales e históricamente planteados, tales como los problemas de discriminación racial, pobreza y género; así mismo las condiciones relacionadas con el hábitat inmediato: precariedad de servicios de saneamiento público, vivienda, acceso a servicios de salud, al igual que la alta incidencia de enfermedades respiratorias [9].

\section{C.Estrategias utilizadas para la atención de la salud mental infantil}

La UNICEF, como organismo de las Naciones Unidas encargado de los problemas de la infancia y la adolescencia, ha solicitado a los gobiernos del mundo la incorporación de una estrategia específica de salud mental y bienestar psicológico en los planes de reconstrucción, así como asumir la salud mental como un elemento transversal en todas las áreas de actuación. De la misma manera, se ha orientado a detectar cuanto antes el riesgo en la salud mental de ese segmento de la población, y fortalecer el servicio de atención de salud mental y apoyo psicosocial con carácter permanente [2].

Durante la infancia y la adolescencia, el desarrollo cognitivo, emocional y social, atraviesa sus fases más cruciales. Por ello, las organizaciones internacionales han asumido la integración de los servicios de salud mental con el apoyo psicosocial. Esta acción es multidisciplinaria y se guía por los siguientes lineamientos generales: promover la salud mental, prevenir las condiciones que pueden afectar a la salud mental, proteger los derechos humanos y diagnosticar, atender y tratar a los niños, niñas y adolescentes, así como a sus cuidadores cuando tienen problemas de salud mental.

La suspensión de las clases ha tenido como consecuencia la diminución del apoyo social proveniente de la escuela. No sólo se ve afectado el aprendizaje y las actividades escolares específicamente, sino también la esfera comunitaria, pues se reduce el acceso a recursos de salud, servicios para la defensa de los derechos de los niños, niñas y adolescentes, y control del abuso y la violencia doméstica. Por otra parte, se ha aumentado el estrés de los padres y los efectos conductuales de las restricciones en las actividades diarias, relacionales y 
de circulación. Se ha observado también un aumento en los conflictos interpersonales lo cual conlleva un incremento de episodios de violencia contra los niños [19].

Todo esto ha conllevado al replanteamiento de acciones locales organizadas a partir de las demandas de los territorios específicos y sus habitantes. El objeto de las acciones del cuidado se convierte en el binomio existencia-sufrimiento; la premisa teórico conceptual es el campo multidisciplinario; y las intervenciones mismas se basan en la diversidad de estrategias y la inventiva. El agente de la atención se encuentra en el colectivo; el lugar de la atención se configura en una red guiada por la intersectorialidad y la práctica social se debe orientar hacia la inclusión y la solidaridad [20].

La UNICEF ha divulgado algunos consejos para los padres y madres, como las personas sobre las cuales descansa una gran responsabilidad para resguardar la salud integral especialmente la mental de sus hijos. En primer lugar, se llama la atención acerca de algunos signos y síntomas de que la salud mental de los niños está siendo afectada por el confinamiento. Estos pueden ser dificultades para dormir, dolor de estómago, mal humor, agresividad, irritabilidad o tener ataques de miedo y hasta de pánico. Frente a ello, lo primero que se recomienda es conversar con el niño y explicarle que es normal sentir miedo y que la situación es temporal y que puede pasar pronto. En este sentido de mantener una comunicación fluida con los hijos, se indica que es importante escuchar a los niños, no ocultarles información, tratar de usar un lenguaje sencillo, honesto y apropiado para su edad.

Hay que reforzar la idea de que estar en casa brinda una importante protección a todo el grupo familiar. Al mismo, se recomienda limitar el uso de las redes sociales y los medios de comunicación masiva en general, pues una sobreexposición a la información incrementa el miedo y el estrés, aparte de los contenidos falsos que abundan por esos canales. Para los adultos es fundamental conservar la calma, para así comunicársela a los hijos. Además, es importante que los niños no permanezcan más de una hora sin movimiento; frente a esto hay que planificar realizar algún tipo de actividad física en grupo, como bailar, hacer gimnasia o desarrollar algún juego donde se muevan. Hay que tomar en cuenta que los juegos son una parte importante de la vida de los niños, por ello se recomienda participar en ellos o inventar algunos donde puedan interactuar todos los miembros del grupo familiar [21].

También es recomendable establecer rutinas para brindar estructura a los niños, y crear un ambiente de nueva normalidad que beneficie, tanto a los pequeños, como a los adultos. Hay que establecer horarios para las comidas, jugar, estudiar, ver películas, leer y alguna otra actividad. Hay que evitar recurrir a la violencia verbal o física para controlar las situaciones con los niños y niñas. También es muy recomendables practicar con los hijos las medidas higiénicas básicas, como es el lavado de las manos y demás, como la limpieza y el arreglo diario de la casa [22].

Algunas instituciones gubernamentales han editado textos con cuentos y versos para niños acerca de la pandemia que, en un lenguaje divertido, en rima, explican al público infantil, lo que está pasando con la pandemia de la COVID 19 y las medidas sanitarias que deben realizar para cuidarse a sí mismos y a sus hermanitos menores: lavarse las manos, estornudar tapándose boca y nariz con el antebrazo, entre otras.

Por otra parte, se han divulgado instrucciones y recomendaciones dirigidas a los adultos que tienen a su cargo familias con niños, niñas y adolescentes. En este se insta a los adultos a mantenerse física y emocionalmente activo y saludable, procurar disponer de un espacio y un tiempo para sí mismo, compartir las inquietudes y temores con otros adultos en quienes puedan apoyarse y hasta recibir tranquilidad y calma, no exponerse a información indiscriminada. Estar pendiente de verificar las fuentes y que estas sean confiables. Regular y gestionar las emociones, considerando que es normal sentir preocupación y hasta tener la sensación de estar desbordados por la situación. Al sentirse mejor, se ayuda a los hijos a afrontar los desafíos emocionales de la pandemia [23].

En este contexto, es fundamental comprender la importancia del mensaje tranquilizador para los hijos que estimule el apego, contextualizado en el vínculo amoroso parentofilial, signado por un amor incondicional. El fundamento teórico de esta recomendación es la teoría del apego que plantea que el ser humano desde su nacimiento necesita desarrollar una relación con al menos un cuidador principal con la finalidad que su desarrollo social y emocional se produzca con normalidad. Quiere decir que el establecimiento de este primer vínculo fundamenta la seguridad del niño, pero también signa la futura seguridad del adulto, por lo tanto, demarca la dinámica a largo plazo de las relaciones con otros seres humanos. El apego se centra en la interacción entre, principalmente, madre e hijo, o cualquier adulto mayor colocado en el lugar de protector. No solamente es por cubrir la necesidad del bebé, sino que son los adultos que se ubican en ese lugar, adultos sensibles y receptivos a las relaciones sociales y que permanecen como cuidadores consistentes durante el periodo que va desde aproximadamente los seis meses a los dos años de edad, aunque después el vínculo continúa el resto la vida [24].

La profunda incondicionalidad amorosa se muestra desde la parentalidad. Son los padres que se ofrecen como protectores incondicionales de los hijos y no a la inversa. Esta modalidad de búsqueda en figuras de apego se acciona toda la vida. Esto quiere decir que las figuras de apego no solo están en nuestra infancia. Esa necesidad de sentirse protegido y socorrido afectivamente en la infancia, se sistematizará toda la vida. Estos tutores pueden ser imaginarios o reales, pueden ser padres y madres, abuelos y abuelas, tíos y tías queridos, vecinos, parejas, profesores; o también pueden ser no humanos como novelas, frases, películas, libros, publicidades o solamente una sonrisa o una palabra que de confianza que constituya una muestra de amor y nos motive a resurgir. Los profesionales de la salud podemos también entrar en esta categoría. Por tales razones, las explicaciones que los padres o cuidadores principales proporcionan a los niños, niñas y adolescentes son una medida de protección y expresión de afecto, no solamente por su contenido y su forma de expre- 
sar el mensaje, sino por la función que desarrollan, o sea la de tutores de resiliencia que, como figuras de apego, otorgan seguridad por el lugar que ocupan para los más jóvenes y pequeños [17] [25].

Lo importante es comunicar a los hijos seguridad y confianza, incluso informándole la verdad sobre algún familiar o conocido que haya sido infectado, evitando, al mismo tiempo, involucrar a los pequeños en conversaciones de adultos sobre el tema. Hay que tener presente que el exceso de información y preocupación los lleva a sentirse vulnerables y hasta atemorizados [26]. Hay que expresar atención a sus demandas, preguntar cómo se sienten, no invalidar sus sentimientos. Desarrollar actividades junto a ellos como dibujar o jugar. Los adultos deben saber diferenciar sus propios temores y preocupaciones de los de sus hijos pequeños. Hacer sentir que la familia, como un equipo, está afrontando con seguridad la situación, tomando en cuenta de que es inevitable que los pequeños reciban información por otras vías. En lugar de insistir en la represión y el control en el uso de las tecnologías, orientar el uso de ellas, hacia chats, videos y demás recursos adecuados, con información adecuada y confirmada, además que contribuyan a mantener un clima emocional relajado y seguro. Así mismo, es importante establecer un conjunto de normas y limitaciones, que incluyan las medidas sanitarias generales, como el lavado de las manos y las disposiciones ante los estornudos, y demás [27] [21].

Diversos estudios han mostrado la eficacia de las estrategias metacognitivas en el mejoramiento del afrontamiento del estrés infantil. Una adecuada información, brindada con un lenguaje familiar y con una actitud calmada y seria, ayuda eficazmente al niño, niña o adolescente a disponer de estrategias para afrontar situaciones de estrés propias de la actual pandemia [28].

\section{Metodología}

\section{A.Revisión documental}

La metodología empleada en el presente artículo es el de la revisión documental. Se trata de un proceso de búsqueda, recuperación, análisis, crítica e interpretación de datos secundarios, es decir, los obtenidos y registrados por otros investigadores en fuentes documentales impresas, documentales, audiovisuales o electrónicas [29]. En este artículo, se utilizaron fuentes como artículos en revistas científicas, tesis de grado, publicaciones oficiales y de organismos internacionales de la salud.

Especial atención tuvieron los documentos oficiales de las organizaciones internacionales, donde se plasman las orientaciones generales más importantes para la atención mental de los niños, niñas y adolescentes de los sectores más vulnerables.

Para llevar a cabo la búsqueda, se procedió a realizar una exploración en las bases de datos de revistas científicas como REDALYC y PUBMED, como redes destacadas en la divulgación de resultados de investigaciones en América Latina. Dada la abundancia de material, se consideró en próximas in- vestigaciones explorar otras redes de información. Para realizar la búsqueda, en primer lugar, se desglosaron los términos claves de los enunciados conocidos del problema a investigar; luego se desarrolló una estrategia de búsqueda mediante operadores de intersección (and, or, not), colocando frases en comillas o recuperando variantes de términos.

Se distinguieron y agruparon las fuentes, según las categorías de: artículos científicos, libros o tesis de grado. A continuación, se procedió a la lectura y captación de las nociones y conceptos principales, plasmadas tanto en los resúmenes como en el cuerpo de las publicaciones. Igualmente, se registraron los datos, informaciones y explicaciones obtenidas en el plano teórico y en el referido a experiencias.

\section{Resultados}

\section{A.Pandemia y cuarentena: riesgos de salud mental de los ni-} ños

La documentación revisada confirma que la pandemia por COVID 19, así como las propias medidas profilácticas que se han aplicado en todo el mundo, son factores de riesgo para la salud mental de los niños, niñas y adolescentes.

Los síntomas más comunes de que la salud mental de este segmento de la población está siendo afectado, son: la ansiedad, los trastornos del sueño, depresión, los miedos y pánicos, así como la irritabilidad y las actitudes agresivas. Estas señales de afectación se notan más en aquellos niños que no han adquirido habilidades de afrontamiento de situaciones de estrés y resiliencia. Especialmente, los infantes de sexo masculino que, además, arrastran problemas emocionales desde sus hogares por conflictos familiares y poca comunicación con los padres. El confinamiento puede dar pie también a algunas situaciones que afectan la salud mental de los niños, niñas y adolescentes, porque ellos manifiestan tristeza e incluso depresión y ansiedad, cuando ello significa también el alejamiento de otros miembros de la familia y a las amistades de su entorno.

El cierre de las escuelas es un importante factor estresante en los niños, niñas y adolescentes, pues interrumpe el proceso de aprendizaje, así como las rutinas necesarias para estructurar la cotidianidad de los sujetos menores. Así mismo, el cese de las relaciones diarias con sus compañeros de edad, puede repercutir en alguno de los síntomas de afección emocional.

Los estudios empíricos revelan que las instrucciones para el relajamiento, así como una buena información comunicada de manera serena y sencilla, apropiada para su edad, pueden contribuir a desarrollar habilidades para el afrontamiento del estrés propio de la situación. Esto se advirtió especialmente en las niñas, mientras que los niños tendían a adoptar actitudes agresivas inadecuadas.

\section{B.Vulnerabilidades de niños, niñas y adolescentes}

Los niños y jóvenes que ya tenían dolencias mentales ven exacerbados sus síntomas. Igualmente, aquellos menores que viven en familias con problemas de abuso o violencia doméstica, muy asociados con las carencias económicas y de 
marginamiento social de las familias.

Los efectos de la pandemia en la salud mental de los niños, niñas y adolescentes son más extendidos e importantes en aquellos sectores sociales más desfavorecidos. Ellos resienten los problemas en el hogar, magnificados por el confinamiento, ya que las consecuencias sociales y económicas son más patentes en el estrés que sufren los adultos por problemas de empleo y manutención de la familia. Por ello, tanto los organismos internacionales como algunos gobiernos, han integrado la atención psicosocial a los servicios de salud mental.

\section{C.Estrategias de afrontamiento necesarias ante riesgos de salud mental}

Los organismos internacionales han dado lineamientos para acompañar la acción profiláctica con medidas de tipo social y económico, debido a que el sector de la población más vulnerable a los efectos de la pandemia en lo que se refiere a la salud mental, se ubica en aquellos grupos más desfavorecidos desde el punto de vista socioeconómico,

En la asunción de estrategias de afrontamiento ante los riesgos de la salud mental, se ha resaltado el rol que adquieren los padres en la tarea de preparar a los niños, niñas y adolescentes. Para ello, algunos gobiernos han publicado guías de acción e incluso libros de cuentos, en las cuales se incluyen explicaciones amenas, especialmente dirigidos a niños, así como recomendaciones acerca del estilo de comunicación y diálogo que debe privar hacia los pequeños. Igualmente, la planificación de actividades lúdicas en conjunto con toda la familia, la cual debe aparecer como un equipo que enfrenta la situación.

\section{Conclusiones}

A.La pandemia del COVID 19 y sus efectos en la salud mental de niños, niñas y adolescentes, es un factor adicional en un complejo de problemas sociales

Deben estudiarse los efectos de la pandemia CODIV 19 en toda su complejidad que abarca muchos más aspectos que la extensión de infección y la curación de los enfermos. Esa complejidad de problemas atañe a la situación de marginamiento y precarización social y económica de amplios segmentos de la población, así como problemas de salud en general y de la mental en especial, que se vienen trayendo desde hace ya muchos años y que, ahora con la pandemia, se agudizan.

La salud mental es otro de los aspectos más resaltantes de esa complejidad de problemas. Los niños, niñas y adolescentes son el sector más vulnerable se ven confinados en el hogar, interrumpida su vida escolar, las relaciones con sus amigos y compañeros de escuela, así como son impactados por información descontrolada o inadecuada que circula por las redes sociales y los medios de divulgación en general.

B.Los niños, niñas y adolescentes deben ser preparados en estrategias de afrontamiento y desarrollo de resiliencia ante el riesgo de salud mental

A los padres y adultos en general les toca asumir la responsabilidad de crear las condiciones propicias para enseñarles estrategias de afrontamiento psicológicas y resiliencia basada en el apego afectivo. Se recomienda entrenar a los niños en técnicas de relajación, así como estructurar la cotidianidad en actividades donde participe toda la familia (juegos, labores de limpieza) y mantener las relaciones interpersonales en el mejor nivel posible.

Las familias deben disponer apoyos comunitarios y sociales para poder desempeñar esa labor que refuerce los pilares de las relaciones familiares. En esa red de apoyo debe incorporarse la totalidad de la comunidad y los servicios públicos de salud y de desarrollos comunitarios locales.

En el interior del hogar, es muy importante la comunicación abierta, el lenguaje sencillo y adecuado y la disposición de la información lo más amplia posible, sin reproducir los falsos bulos que se distribuyen en las redes sociales,

\section{C.Hacen falta estudios empíricos multidisciplinarios.}

Es importante realizar nuevos estudios de campo, en los cuales se combinen metodologías cuantitativas y cualitativas, para determinar y diagnosticar los efectos y el alcance de los riesgos de la salud mental, así como apreciar las narraciones de los comportamientos de las familias y los entornos sociales donde se desenvuelven los más pequeños, para poder observar el estilo de la comunicación y de las relaciones interpersonales en general.

Esos estudios deben ser multidisciplinarios, pues los impactos de la pandemia van mucho más allá que el aspecto corporal o biológico, para extenderse a la economía de las familias, las relaciones sociales, el funcionamiento de los grupos familiares, el acceso a los servicios públicos como privados de atención a la salud.

\section{Referencias}

[1]Organización de las Naciones Unidas para la Infancia, «Plataforma de la Infancia. Convención de los derechos de la infancia,»16 Diciembre 1998. [En línea]. Available: https:// plataformadeinfancia.org/derechos-de-la-infancia/?gclid_ Cj0KCQiAx9m. [Último acceso: 30 enero 2021].

[2]UNICEF, salud mental e infancia en el escenario de la COVID 19, Madrid: UNICEF, 2020.

[3]ANPIR, «ANPIR. Salud mental en la era del confinamiento del COVID 19: posicionamiento de las asociaciones de psiquiatría y psicología clínica especialmente en niños y adolescentes,» 10 mayo 2020. [En línea]. Available: hettops:// www.anpir.org/salud-,ental-en-la-era-del-confinamiento-posicionamiento-de-las-asociaciones-de-psiquiatría-y-psicologia-clinica-especialmente-en-niños-y-adolescentes. [Último acceso: 30 enero 2021].

[4]R. Paricio del Castillo y M. Pando Velasco, «Salud mental infantojuvenil y pandemia COVID 19: cuestiones y retos,» Revista de Psiquiatría Infantojuvenil, vol. 37, nº 2, pp. 30-44, 2020.

[5]J. Rivera Baños, «Invetigación sobre estrés infantil,» Re- 
vista de Ciencias Sociales y Humanidades, vol. 2, $\mathrm{n}^{\mathrm{o}}$ 2, pp. 62-83, 2013.

[6]C. Wang, R. Pan, X. Wan, Y. Tan y L. Xu, «Inmediate psychological responses and asociated factors during the initial stage of the COVID 19 epidemic among the general population in Cgina,» 26 abril 2020. [En línea]. Available: https:// doi.org/10.3390/ijerph17052172. [Último acceso: 16 enero 2021].

[7]M. Zayas Fajardo, R. Román López y L. R. L. Y. Rpdríguez Zayas, «Repercusión psicológica en niños y adolescentes y la familia relacionada con el aislamiento social por la COVID 19,» Revista Dr. Zoilo Marinello, vol. 46, n 1, pp. 12-20, 2021.

[8]A. Martínez Taboas, «Pandemia, COVID 19 y salud mental ¿Qué sabemos actualmente?,» Revista Caribeña de Psicología, vol. 4, no 2, pp. 7-17, 2020.

[9]O. Galindo Vásquez y M. Ramírez Orozco, «síntomas de ansiedad, depresión y conductas de autocuidado durante la pandemia COVID 19,» Gaceta Médica, vol. 15, nº 6, pp. 298305, 2020 .

[10]G. Garrido y G. González, «¿La pandemia COVID 19 y las medidas de confinamiento aumentan el reisgo de violencia hacia los niños, niñas y adolescentes,» Archivos de Pediatría , vol. 91, nº 4, pp. 194-198, 2020.

[11]R. Jerves Mora, «Pandemia y ansiedad social,» Revista de la Facultad de Ciencias Médicas de la Universidad de Cuenca, vol. 4, nº 6, pp. 13-16, 2020.

[12]J. Cifuentes Faura, «Consecuencias del cierre de las escuelas por el COVID 19 en las desigualdades educativas,» Revista Internacional de Educación para la Justicia Social, vol. $9, \mathrm{n}^{\circ} 3$, pp. 10-22, 2020.

[13]X. Méndez, J. Ortigosa y S. Pedroche, «Preparación para la hospitalización infantil y el afrontamiento del estrés,» spciología Conductual, vol. 4, nº 2, pp. 193-209, 2015.

[14]J. Palacio Ortiz y J. Londoño Herrera, «Trastornos psiquiátricos en niños, niñas y adolescentes en tiempos de pandemia por COVID 19,» Revista Colombiana de Psicología, vol. 1, no 2, pp. 21-29, 2020.

[15]Inter Agency Standing Comitee, «iasc: reference group mental health and psychosocial support emergency settings,» 8 mayo 2020. [En línea]. Available: https:// interagencystandingcomitee.org/iasc-reference-group-mental-health-and-psychosocial-support-emergency-settings/interim-briefing. [Último acceso: 30 enero 2021].

[16]Harvard University, «Operational Guideline developping child,» 13 2020. [En línea]. Available: https://developpingchild.harvard.edu/science/jey-concept/resilience. [Último ac- ceso: 23 enero 2021].

[17]M. Rodríguez Ceberio y C. Calligaro, «Instrucciones a niños, niñas y adolescentes sobre la pandemia de la COVID 19. Contenidos y formas de comunicación.,» Revista Prospectivas en Psicología, vol. 5, no 1, pp. 4-14, 2020.

[18]F. Morales Rodríguez, M. Trianes Torres y J. Miranda Páez, «Diferencias por sexo y edad en el afrontamiento infantil del estrés cotidiano,» EJREP, vol. 10, n² 26, pp. 95-110, 2012.

[19]M. Cid, A. Fernández y G. Morato, «Atención psicosocial y la pandemia,» Journal of Education Researchs, vol. 10, $\mathrm{n}^{\mathrm{o}} 2$, pp. 178-201, 2020.

[20]M. Barboza, A. Souza, G. García Morato y M. Morais, «Atención psicosocial y pandemia COVID 19: reflexiones en la atención a la infancia y adoelscencia que viveen contextos socialmente vulnerables,» Multidisciplinary Journal of Educational Research, vol. 10, no 2, pp. 178-201, 2020.

[21]L. Del Moral y C. Larkins, «Construyendo el arco iris: apoyando la participación de niños, niñas y adolescentes en la configuración de respuestas al COVID 19,» Sociedad e Infancia, vol. 4, $\mathrm{n}^{\circ}$ 1, pp. 275-277, 2020.

[22]UNICEF, «Consejos para papá y mamá. Apoyo emocional para niños y niñas ante la COVID 19,» UNICEF- Gobierno de México, México, 2020.

[23]L. Dalton, E. Rapa y A. Stein, «Protecting the psychological health of childrem through effective communication about COVID 19,»12 Agosto 2020. [En línea]. Available: https://doi.org/10.1016/S2352-4642(20)30097-3. [Último acceso: 26 Enero 2021].

[24]J. Bowlby, «Separation anxiety,» International Journal of Psychoanalysis, vol. 41, nº 2, pp. 89-113, 1969.

[25]N. Cedeño, M. Cuenca y A. Mojica, «Afrontamiento del COVID 19: estrés, miedo, ansiedad y depresión,» Enfermería investiga, vol. $5, \mathrm{n}^{\circ} 3$, pp. 63-70, 2020.

[26]B. Cyrulnik, Los patitos feos. La resiliencia: una infancia infeliz no determina la vida, Madrid: Gedisa, 2008.

[27]SISS-UNICEF, «SISS Chile. ¿Cómo proteger el bienestar emocional de los niños y niñas frente a la crisis generada pro el COVID 19?,» 12 marzo 2020. [En línea]. Available: www.SISS.gob.cl. [Último acceso: 30 enero 2021].

[28]C. Valiente Barroso, M. Martínez, P. Cabal García y J. Alvarado, «estrés infantil, estrategia de aprendizaje y motivación académica: un modelo estructural,» revista de Psicología y Educación, vol. 15, nº 1, pp. 46-66, 2020.

[29]F. Arias, el proyecto de investigación. Introducción a la metodología científica, Caracas: Episteme, 2012. 